\title{
Epidemiology of Superficial Fungal Infections in Hospital Settings in Togo and Senegal from 2019 to 2020
}

\author{
Minlekib PC ${ }^{1}$, Dorkenoo $\mathrm{AM}^{2}$, Sow $\mathrm{D}^{3 *}$, Dia $\mathrm{M}^{1}$, Manga IA ${ }^{1}$, Fall CB ${ }^{1}$, Lelo $\mathrm{S}^{1}$, \\ Sossou $\mathrm{E}^{2}$, Sylla $\mathrm{K}^{1}$, Ndiaye $\mathbf{M}^{1}$, Ndiaye $\mathrm{JL}^{1}$, Tine $\mathrm{RC}^{1}$, Dieng $\mathrm{T}^{1}$ and Faye $\mathrm{B}^{1}$ \\ ${ }^{1}$ Department of Parasitology-Mycology, University Cheikh Anta Diop, Senegal \\ ${ }^{2}$ Department of Biological and Fundamental Sciences, University of Lome, Togo \\ ${ }^{3}$ Department of Parasitology-Mycology,University Gaston Berger, Senegal
}

*Corresponding author: Doudou Sow, Department of Parasitology-Mycology, Université Gaston Berger, Saint- Louis, Sénégal, Tel: 775425028 (221); Email: doudou.sow@ugb.edu.sn

\section{Research Article \\ Volume 4 Issue 2}

Received Date: November 12, 2021

Published Date: November 29, 2021

DOI: $10.23880 /$ oajmms-16000149

\section{Abstract}

Background: Superficial fungal diseases are cosmopolitan affections caused by yeast and filamentous microscopic fungi (dermatophytes and moulds). Their very high prevalence and worldwide distribution make them one of the most common dermatological diseases. Our study aims to describe the profile of superficial fungal diseases diagnosed in Senegal and Togo and to identify the causative agents circulating in both countries.

Material and Methods: This is a descriptive study carried out in Senegal and Togo, involving 602 and 135 patients respectively. The patients presenting clinical signs were screened in the Parasitology-Mycology laboratories of the Fann University Hospital in Dakar (from February 2019 to February 2020) and Sylvanus Olympio University Hospital in Lomé (from October 2019 to March 2020). Samples collected were examined using routine mycological techniques (Direct examination and culture).

Results: In Senegal 293 (48.67\%) out of 602 patients enrolled were diagnosed with superficial fungal diseases. Patients under 10 old years and female, most affected, represented $23.42 \%$ and $61.39 \%$ of cases respectively. The patients were seen mostly in September with 27 positives cases out of 42 . Trichophyton soudanense and Candida albicans complex were the most common species, with a prevalence rate of $30.37 \%$ and $26.96 \%$ respectively. In Togo, $67.41 \%$ (91) of the 135 patients presented superficial mycosis. The female (58.76\%) and the patients aged 31-40 year old (21.65\%) were the most affected. Highest prevalence is recorded in October with 11 positive cases out of 13. Malassezia furfur was the most common species with a prevalence of $28.57 \%$. The same species of fungi were found in both countries with the exception of three fungi Trichophyton violaceum, Aspergillus candidus (respectively 3 cases and 1 case in Senegal) and Trichophyton tonsurans ( 3 cases in Togo).

Conclusion: Superficial mycoses are a frequent reason for consultation in Senegal and Togo. Dermatophytes and yeasts of the genus Candida and Malassezia are the most common agents involved. The use of innovative diagnostic techniques could allow a more precise identification of species for a better therapeutic management.

Keywords: Superficial Fungal Infections; Hospital; Senegal; Togo

\section{Introduction}

Superficial fungal diseases are cosmopolitan affections caused by yeast and filamentous microscopic fungi (dermatophytes and moulds). Their very high prevalence and worldwide distribution make them one of the most common dermatological diseases [1]. Nearly one billion people have skin fungal diseases, which makes this disease only slightly 


\section{Open Access Journal of Mycology \& Mycological Sciences}

less common on the planet than headaches and dental caries [2]. They characterize several symptoms including skin, nail and hair disorders. These symptoms are very unpleasant because they not only modify the aesthetics of the affected persons but also create discomfort and therefore affect their quality of life. Laboratory diagnosis of superficial mycoses is based mainly on direct examination of the sample followed by culture.

The distribution of the dermatomycoses, their aetiological agents and the predominating anatomical infection patterns vary with geographical location and a wide range of environmental and cultural factors [3]. Currently, Trichophyton rubrum, Trichophyton interdigitale, Trichophyton tonsurans, Microsporum canis, Malassezia spp. and Candida spp. are the main fungal species identified in superficial mycoses [4,5]. Recent studies, in Senegal, have reported predominance of Trichophyton soudanense $(24,9 \%)$ and Trichophyton rubrum (13,7\%) among dermatophytes, Candida albicans (26,9\%) and Candida sp. (6,6\%) among yeasts, Fusarium solani $(2,7 \%)$ and Fusarium oxysporum $(0,9 \%)$ among moulds [6]. Most of the epidemiological studies in Africa are conducted using sometimes different laboratory procedures. It is therefore quite important to standardize the techniques used in order to better describe the profile of causative agents circulating in different settings. Our study aims to update the epidemiology of superficial mycoses and the profile of the circulating causative agents in Togo and Senegal.

\section{Materials and Methods}

\section{Study area and Patients}

This descriptive, prospective study was carried out at two sites: the Parasitology-Mycology laboratories of the Fann University Hospital (SENEGAL) between February 2019 and February 2020; and the Sylvanus Olympio University Hospital (TOGO) between October 2019 and March 2020. All the patients screened during the study period were enrolled.

\section{Samples Collection}

Hair, nail or skin fragments from all patients with suspected superficial mycosis (Tinea capitis, tinea ungium, tinea corporis or tinea versicolor) were sampled.

The removal of each lesion was carried out separately with sterile material, before or at distance from any antifungal treatment. Also, each patient was asked to wash the affected areas with ordinary soap and to protect them either with a cap (for ringworm of the scalp) or a sock (for intertrigos, keratodermias, and onychomycosis) before the sampling.

\section{Tinea capitis}

After an examination in Wood's light, the area of alopecia was removed using a scalpel blade and the fluorescent hair was removed using tweezers.

\section{Tinea ungium}

The sampling was preceded by cleaning with Dakin and scraping the nail table to remove the superficial part. Then the nail fragments and flakes were collected with a scalpel blade.

\section{Tinea corporis}

The sampling started by cleaning with Dakin. Then a scraping on the periphery of the lesion was performed with a scalpel blade to collect the scales.

\section{Tinea versicolor}

A piece of tape was applied to the suspect lesion and placed on a microscopy slide.

\section{Mycological Diagnosis}

It was carried out with strict adherence to the following four steps: Direct Examination, Culture and Identification.

\section{Direct Examination}

Skin scraps, hairs, nails fragments were placed on a microscopy slide with few drops of $30 \%$ aqueous potassium hydroxide $(\mathrm{KOH})$ and then covered with a slide. The observation was made under a microscope with $\mathrm{x} 10$ and $\mathrm{x} 40$ objectives looking for hyphae, isolated or "grape bunch" yeast.

\section{Culture and Identification}

Different samples were placed at several points on the surface of Sabouraud Chloramphenicol (SC) and Sabouraud Chloramphenicol plus cycloheximide (SCC) media at 25 $\mathrm{C}$ for up to 4 weeks for the culture. The identification of fungal species was done based on their cultural caracteristics including color of the colonies, their shape, relief, consistency, size and looked for the presence of pigment diffusing in the agar. Some particular aspects of dermatophytes were also researched microscopically such as hyphae appearance, presence of chlamydospores, microconidies and macroconidies [7].

The yeasts were identified based on morphological and physiological (germination test) characteristics. The 


\section{Open Access Journal of Mycology \& Mycological Sciences}

interpretation of the yeasts and moulds was based on the results of direct examination and the abundance of the colonies [8].

\section{Ethical Considerations}

This study has been approved by the Ethics Committee of the Cheikh Anta Diop University of SENEGAL (Reference number: 0413/2019/CER/UCAD) and by the Bioethics Committee for Health Research of TOGO (Reference number: 19/2020/CBRS).

\section{Statistical Analysis}

All the data collected from this study were entered into Excel and analyzed using Epi-info 7.2 software. Quantitative data are presented in averages and qualitative data in numbers and percentages.

\section{Results}

A total of 602 patients in Senegal and 135 in Togo were sampled. In Senegal we collected 677 samples of which the most common infection site was scalp with $307(45,34 \%)$ of the total cases followed by nail fragments $204(30,13 \%)$ and skin scales 166 (24,51\%). In Togo we collected 143 samples and skin scales was the most common infection site 93 $(65,0.3 \%)$ followed by nail fragments $41(28,67 \%)$ and scalp 9 (6,29\%). $293(48,67 \%)$ and $91(67,41 \%)$ were confirmed to have superficial mycoses respectively in Senegal and Togo. The mean age was 31.21 years (range 0.5-89 years) in Senegal and 32.93 years (range 0.16-75 years) in Togo. Most cases were between 0 and 10 years of age $(17,17 \%)$ in Senegal and 31 to 40 years of age $(22,96 \%)$ in Togo (table 1 ). In both countries, women have been represented more than men, with frequencies of $64.95 \%$ in Senegal and $60 \%$ in Togo (Table 1).

\begin{tabular}{|c|c|c|c|c|}
\hline & SENEGAL & \multirow{2}{*}{$\%$} & TOGO & \multirow{2}{*}{$\%$} \\
\hline & Number & & Number & \\
\hline \multicolumn{5}{|l|}{ SEX } \\
\hline Female & 391 & 64,95 & 81 & 60 \\
\hline Male & 203 & 33,72 & 52 & 38,52 \\
\hline No precise & 8 & 1,33 & 2 & 1,48 \\
\hline Total & 602 & 100 & 135 & 100 \\
\hline \multicolumn{5}{|c|}{ Age Group (Year) } \\
\hline $0-10$ & 107 & 17,77 & 9 & 6,67 \\
\hline 20-Nov & 64 & 10,63 & 25 & 18,52 \\
\hline $21-30$ & 100 & 16,61 & 25 & 18,52 \\
\hline $31-40$ & 81 & 13,46 & 31 & 22,96 \\
\hline $41-50$ & 66 & 10,96 & 20 & 14,81 \\
\hline More than 50 & 101 & 16,78 & 19 & 14,07 \\
\hline No precise & 83 & 13,79 & 6 & 4,44 \\
\hline Total & 602 & 100 & 135 & 100 \\
\hline \multicolumn{5}{|l|}{ Sampling Type } \\
\hline Scalp & 307 & 45,34 & 9 & 6,29 \\
\hline Nail & 204 & 30,13 & 41 & 28,67 \\
\hline Skin & 166 & 24,51 & 93 & 65,03 \\
\hline - $\quad$ ITO & 96 & 14,18 & 43 & 30,06 \\
\hline - $\quad \mathrm{K}$ & 48 & 7,09 & 2 & 1,40 \\
\hline EP & 13 & 1,92 & 20 & 13,98 \\
\hline PV & 9 & 1,32 & 28 & 19,59 \\
\hline Total & 677 & 100 & 143 & 100 \\
\hline
\end{tabular}

ITO: Intertrigo; K: Keratoderma; EP: Epidermophytosis, TV: Tinea versicolor

Table 1: Socio-demographic and clinical distribution of the population in Senegal and Togo. 
The number of samples and positive cases, in Senegal, varied monthly, with an averages of 46.31 samples and 22.54 positive cases and the most affected month was September with 27 positive cases out of 42 (Figure 1). In Togo the averages of 22.5 samples and 15.17 positive cases were obtained and the month registrated a highest number of positive cases was October with 11 of 13 (Figure 2).

Senegal

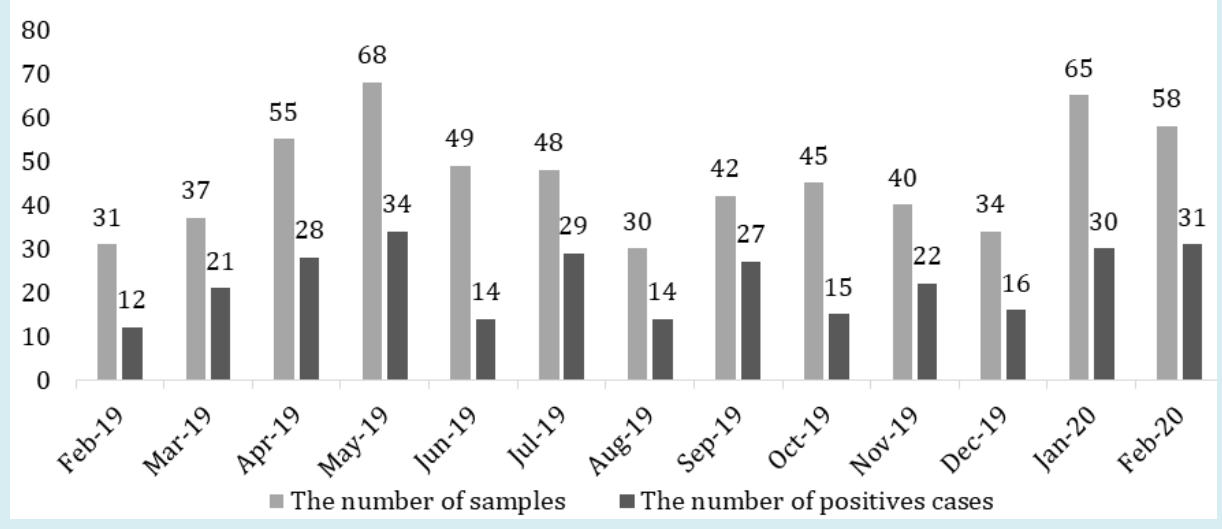

Figure 1: Distribution of population and superficial mycoses according to month of collection in Senegal.

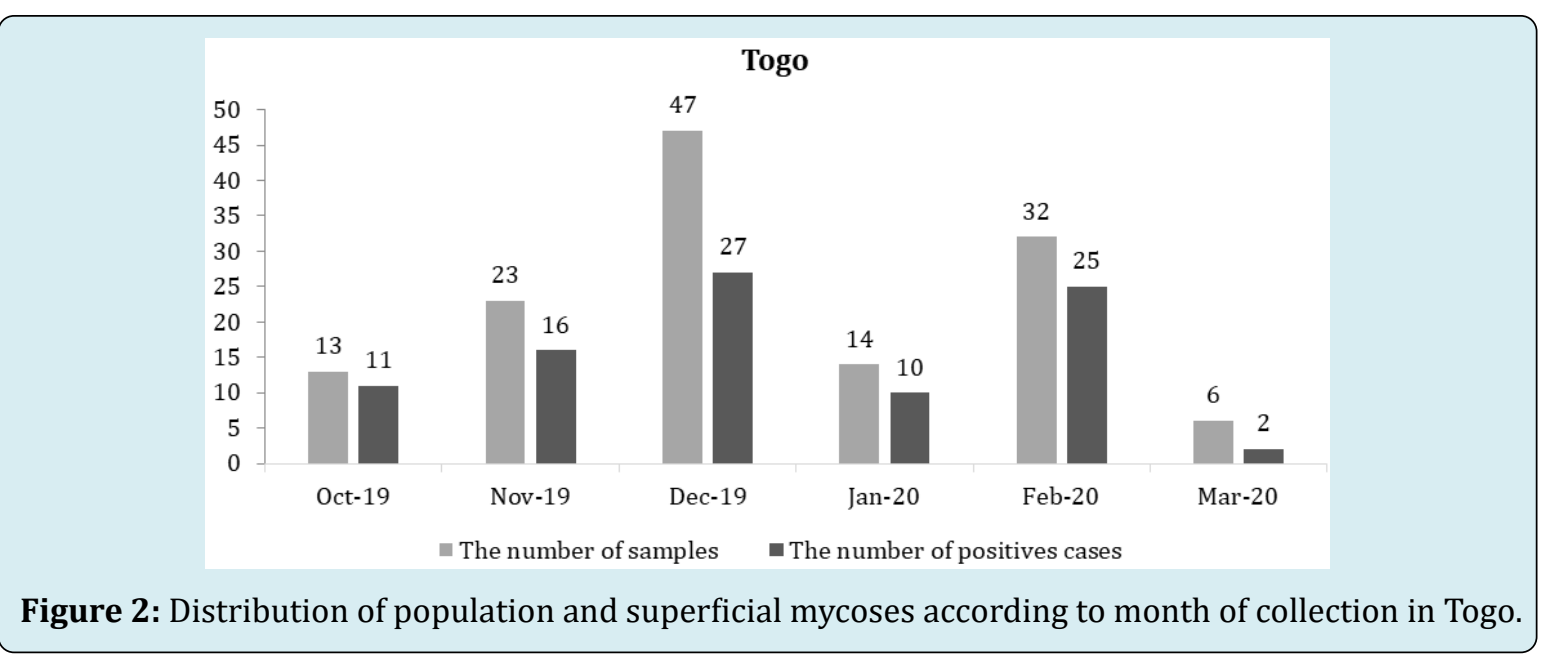

In Senegal, 316 of 677 samples analyzed, were positive with a prevalence of $46.68 \%$. The sampling sites were classified into the following three categories: scalp scales $36.71 \%$ (116), nails fragments $32.91 \%$ (104) and skin scales $30.38 \%$ (96) (Table 2). Overall, 194 (61.39\%) were women (most of cases in scalp: 75 followed by nails: 73 and skin: 46) while $118(37,34 \%)$ were men (Table 2). According to the age group, superficial infections were more frequently encountered in children under 10 years $(n=74)$ followed by adults over 50 years $(n=62)$ and those with age from 31 to 40 years. Most of tinea capitis were found in children under 10 years $(n=65)$ while the cases of tinea ungium $(n=25)$ and tinea corporis $(n=33)$ were common in adults over 50 years old (Table 2).
In Togo, 97 of 143 samples collected, were positive with a prevalence of $67,83 \%$. The sampling sites were classified into the following three categories: skin scales 68, $04(n=66)$, nails fragments 29,9\% (n=29) and scalp scales 2,06\% $(n=2)$ (table 2). Overall, 58,76\% $(n=57)$ were women (most of cases in skin scales : 35 followed by nails: 22) while 37 $(38,14 \%)$ were men (Table 2$)$. According to the age group superficial infections were more frequently found in adults aged 31 to 40 years $(n=21)$ followed by those aged 21 to 30 years $(n=20)$. All of tinea capitis cases were found in children under 10 years $(n=2)$ while most cases of tinea ungium $(n=8)$ were found in adult from 21 and 30 years and most of tinea corporis ( $\mathrm{n}=17)$ in adults from 31 to 40 years old (Table 2). 


\section{Open Access Journal of Mycology \& Mycological Sciences}

\begin{tabular}{|c|c|c|c|c|c|c|c|c|}
\hline & \multicolumn{4}{|c|}{ Senegal } & \multicolumn{4}{|c|}{ Togo } \\
\hline & Scalp & Nails & Skin & Total (\%) & Scalp & Nails & Skin & Total (\%) \\
\hline Total Samples & 307 & 204 & 166 & 677 & 9 & 41 & 93 & 143 \\
\hline Positive Samples & 116 & 104 & 96 & $316(46,68)$ & 2 & 29 & 66 & $97(67,83)$ \\
\hline$\%$ & 36,71 & 32,91 & 30,38 & 100 & 2,06 & 29,9 & 68,04 & 100 \\
\hline \multicolumn{9}{|l|}{ Sex } \\
\hline $\mathrm{F}$ & 75 & 73 & 46 & $194(61,39)$ & - & 22 & 35 & $57(58,76)$ \\
\hline $\mathrm{M}$ & 40 & 29 & 49 & $118(37,34)$ & 2 & 6 & 29 & $37(38,14)$ \\
\hline No precise & 1 & 2 & 1 & $4(0,59)$ & - & 1 & 2 & $3(3,1)$ \\
\hline TOTAL & 116 & 104 & 96 & 316 & 2 & 29 & 66 & 97 \\
\hline \multicolumn{9}{|c|}{ Age group (year) } \\
\hline $0-10$ & 65 & 6 & 3 & $74(23,42)$ & 2 & - & 5 & $7(7,22)$ \\
\hline 20-Nov & 9 & 4 & 4 & $17(5,38)$ & - & 5 & 12 & $17(17,53)$ \\
\hline $21-30$ & 8 & 18 & 12 & $38(12,02)$ & - & 8 & 12 & $20(20,62)$ \\
\hline $31-40$ & 6 & 21 & 16 & $43(13,61)$ & - & 4 & 17 & $21(21,65)$ \\
\hline $41-50$ & 2 & 19 & 21 & $42(13,29)$ & - & 5 & 7 & $12(12,37)$ \\
\hline$>50$ & 4 & 25 & 33 & $62(19,62)$ & - & 6 & 10 & $16(16,49)$ \\
\hline No precise & 22 & 11 & 7 & $40(12,66)$ & - & 1 & 3 & $4(4,12)$ \\
\hline TOTAL & 116 & 104 & 96 & 316 & 2 & 29 & 66 & 97 \\
\hline
\end{tabular}

Table 2: Prevalence of superficial mycoses according to age, sex and sampling site in Senegal and Togo.

In Senegal, 293 patients with single or associated fungus were found, of which $273(93.1 \%)$ isolated and $20(6.82 \%)$ in association. In single infections, T. soudanense was the most common dermatophyte found in 89 or $30.37 \%$ cases (Tinea capitis $=72$, onychomycosis $=3, \mathrm{~K}=3, \mathrm{ITO}=2$, associations $=9$ ) followed by M. langeroniiin 21/7.16\% cases (Tinea capitis=17, Onychomycosis $=1$, associations $=3$ ). Other dermatophyte species were also found such as T. violaceum in 9 cases (Tinea capitis $=7, \mathrm{~K}=1$, associations $=1$ ), . . mentagrophytes in 5 cases (Onychomycosis $=1$, ITO=1, Associations $=3$ ), T. rubrum in 5 cases (Tinea capitis $=3$, associations $=2$ ), T. verrucosum in 3 associations and E. floccosum in one case of ITO. Overall, $C$. albicans/dubliniensis/africana was the most isolated yeast with a prevalence of $26.96 \%$ ( 79 cases), followed by C. sp. with a prevalence of $12.96 \%(\mathrm{n}=38)$, Malassezia furfur $(1.02 \%$, $\mathrm{n}=3)$ and Trichosporon $s p .(0.34 \%, \mathrm{n}=1)$. Among the moulds, Fusarium oxysporum was the most found ( $\mathrm{n}=9, \%=3.07)$ followed by Fusarium $s p$. ( $\mathrm{n}=5,1.70 \%)$, Acremonium $s p .(\mathrm{n}=2$, $0.68 \%$ ) and Aspergillus candidus, Fusarium sp., Neoscytalidium dimidiatum which were found in the same proportions (Table 3). In co-infections, we found associations between dermatophytes ( $T$. soudanense $+M$. langeronii), between yeasts and dermatophytes (T. soudanense associated with $C$. albicans and C. $s p$.), between dermatophytes and moulds ( $T$. verrucosum + F. sp. and F. oxysporum + T. mentagrophytes) and the rest between yeasts and moulds (Table 3 ).

In Togo, 91 patients with single or associated fungus were found including $88(96.70 \%)$ isolated and $3(3.27 \%)$ in associations. In single infections the most common dermatophytes were T. mentagrophytes $(\mathrm{n}=8, \%=8.79$, ITO $=6$, Onychomycosis $=2$ ) and T. rubrum $(\mathrm{n}=8, \%=8.79$, $\mathrm{ITO}=3$, EP=3, Onychomycosis=1, associations $=1$ ). Other species of dermatophytes were found with, in decreasing order: T. soudanense $(\mathrm{n}=3, \%=3.29$, ITO $=2$, association $=1)$, $T$. tonsurans $(\mathrm{n}=3, \%=3.29$, Onychomycosis $=1$, ITO $=1$, $\mathrm{EP}=1)$, $M$. langeronii $(\mathrm{n}=2, \%=2.19$, tinea capitis $=1, \mathrm{EP}=1)$, E. floccosum $(\mathrm{n}=2, \%=2.19, \mathrm{EP}=2)$ and $T$. verrucosum $(\mathrm{n}=1$, $\%=1.09, \mathrm{EP}=1$ ). As far as yeasts are concerned, the following results were obtained in descending order: $M$. furfur $(\mathrm{n}=26)$, C. albicans $(\mathrm{n}=18), C$. $s p .(\mathrm{n}=10)$, Trichosporon $s p .(\mathrm{n}=5)$. The moulds found were Fusarium $s p$. $(\mathrm{n}=1)$ and Neoscytalidium dimidiatum $(\mathrm{n}=1)$ (Table 4). In co-infections we found three associations including T. mentagrophytes $+C . s p .(\mathrm{n}=1), C$. albicans + T. rubum $(\mathrm{n}=1)$ and C. albicans $+A . s p(\mathrm{n}=1)($ Table 4). 


\section{Open Access Journal of Mycology \& Mycological Sciences}

\begin{tabular}{|c|c|c|c|c|c|c|c|c|c|}
\hline \multirow{2}{*}{ Genus } & \multirow{2}{*}{ species } & \multirow{2}{*}{$\begin{array}{c}\text { Tinea } \\
\text { capitis(a) }\end{array}$} & \multirow{2}{*}{$\begin{array}{c}\text { Onycho } \\
\text { mycosis (b) }\end{array}$} & \multicolumn{4}{|c|}{ Tinea corporis } & \multirow{2}{*}{ Co-Infections } & \multirow{2}{*}{ Total (\%) } \\
\hline & & & & ITO (c) & $K(d)$ & EP (e) & PV (f) & & \\
\hline \multicolumn{10}{|l|}{\begin{tabular}{|l|} 
Single Infections \\
\end{tabular}} \\
\hline \multirow{5}{*}{ Trichophyton } & T. mentagrophytes & - & 1 & 1 & - & - & - & $c+d(1) ; b+c(2)$ & $5(1,70)$ \\
\hline & T. soudanense & 72 & 3 & 2 & 3 & - & - & $\begin{array}{c}a+d(1) ; a+b \\
(7) ; a+e(1)\end{array}$ & $89(30,37)$ \\
\hline & T. verrucosum & - & - & - & - & - & - & $\begin{array}{c}\mathrm{c}+\mathrm{d}(1) ; \mathrm{a}+\mathrm{b}(1) \\
\mathrm{a}+\mathrm{e}(1)\end{array}$ & $3(1,02)$ \\
\hline & T. violaceum & 7 & - & - & 1 & - & - & $a+c(1)$ & $9(3,07)$ \\
\hline & T. rubrum & 3 & - & - & - & - & - & $a+d(1) ; a+b(1)$ & $5(1,70)$ \\
\hline Microsporum & M. langeronii & 17 & 1 & - & - & - & - & $a+b(2) ; a+e(1)$ & $21(7,16)$ \\
\hline Epidermophyton & E. floccosum & - & - & 1 & - & - & - & - & $1(0,34)$ \\
\hline \multirow[t]{2}{*}{ Candida } & $\begin{array}{c}\text { C. albicans/ } \\
\text { dubliniensis / } \\
\text { africana }\end{array}$ & - & 44 & 13 & 4 & 2 & - & $\begin{array}{c}d+b(2) ; b+c \\
(10) ; a+c(3) ; \\
a+b+e(1)\end{array}$ & $79(26,96)$ \\
\hline & C. sp. & - & 17 & 10 & 6 & - & - & $\begin{array}{l}b+c(2) ; a+c \\
(1) ; a+b(2)\end{array}$ & $38(12,96)$ \\
\hline Trichosporon & T. sp. & - & 1 & - & - & - & - & - & $1(0,34)$ \\
\hline Malassezia & M. furfur & - & - & - & - & - & 3 & - & $3(1,02)$ \\
\hline Aspergillus & A. candidus & - & 1 & - & - & - & - & - & $1(0,34)$ \\
\hline Acremonium & A. $s p$. & - & - & 2 & - & - & - & - & $2(0,68)$ \\
\hline \multirow{3}{*}{ Fusarium } & F. oxysporum & - & 3 & 6 & - & - & - & - & $9(3,07)$ \\
\hline & F. solani & - & - & 1 & - & - & - & - & $1(0,34)$ \\
\hline & F.sp. & - & 2 & 3 & - & - & - & - & $5(1,70)$ \\
\hline Neoscytalidium & N. dimidiatum & - & - & - & 1 & - & - & - & $1(0,34)$ \\
\hline \multicolumn{2}{|c|}{ Total } & 99 & 73 & 39 & 15 & 2 & 3 & 42 & $273(93,17)$ \\
\hline \multicolumn{10}{|c|}{ Co-Infections } \\
\hline \multicolumn{2}{|c|}{ T. soudanense + M. langeronii } & 1 & - & - & - & - & - & - & $1(0,34)$ \\
\hline \multicolumn{2}{|c|}{ T. soudanense + C. albicans } & - & 1 & - & 1 & - & - & $a+b+d(1)$ & $3(1,02)$ \\
\hline \multicolumn{2}{|c|}{ S. apiospermum + C. sp. } & - & - & - & - & - & - & $b+e(1)$ & $1(0,34)$ \\
\hline \multicolumn{2}{|c|}{ F.sp. + T. verrucosum } & - & - & - & - & - & - & $a+c(1)$ & $1(0,34)$ \\
\hline \multicolumn{2}{|c|}{ F. oxysporum + T. mentagrophytes } & - & - & 1 & - & - & - & - & $1(0,34)$ \\
\hline \multicolumn{2}{|c|}{ F. oxysporum + C. albicans } & - & - & - & - & - & - & $b+c(1)$ & $1(0,34)$ \\
\hline \multicolumn{2}{|c|}{ C.sp. + T. soudanense } & - & 1 & - & - & - & - & $b+c(1)$ & $2(0,68)$ \\
\hline \multicolumn{2}{|c|}{ C. sp. + O.sp. } & - & - & - & - & - & - & $b+c(1)$ & $1(0,34)$ \\
\hline \multicolumn{2}{|c|}{ C. sp. +F.sp. } & - & 1 & - & - & - & - & $c+d(1)$ & $2(0,68)$ \\
\hline \multicolumn{2}{|c|}{ C. sp. + F. oxysporum } & - & - & 1 & - & - & - & - & $1(0,34)$ \\
\hline \multicolumn{2}{|c|}{ C. albicans + F. sp. } & - & 2 & - & - & - & - & $b+c(1)$ & $3(1,02)$ \\
\hline \multicolumn{2}{|c|}{ C. albicans + F. solani } & - & - & - & - & - & - & $c+d(1) ; b+c(1)$ & $2(0,68)$ \\
\hline \multicolumn{2}{|c|}{ C. albicans + A. candidus } & - & - & - & - & - & - & $b+c(1)$ & $1(0,34)$ \\
\hline & tal & 1 & 5 & 2 & 1 & - & - & 11 & $20(6,82)$ \\
\hline
\end{tabular}

ITO: Intertrigo; K: Keratoderma; EP: Epidermophytosis, TV: Tinea versicolor.

Table 3: Distribution of species found in single infections and co-infections depending on the indications in Senegal. 


\begin{tabular}{|c|c|c|c|c|c|c|c|c|c|}
\hline \multirow{2}{*}{ Genus } & \multirow{2}{*}{ Species } & \multirow{2}{*}{$\begin{array}{c}\text { Tinea } \\
\text { capitis (a) }\end{array}$} & \multirow{2}{*}{$\begin{array}{c}\text { Onychomycosis } \\
\text { (b) }\end{array}$} & \multicolumn{4}{|c|}{ Tinea corporis } & \multirow{2}{*}{$\begin{array}{c}\text { Co- } \\
\text { Infections }\end{array}$} & \multirow{2}{*}{ Total (\%) } \\
\hline & & & & ITO (c) & $K(d)$ & EP (e) & TV (f) & & \\
\hline \multicolumn{10}{|l|}{ Single Infections } \\
\hline \multirow{5}{*}{ Trichohyton } & T. mentagrophytes & - & 2 & 6 & - & - & - & - & $8(8,79)$ \\
\hline & T. soudanense & - & - & 2 & - & - & - & $\mathrm{c}+\mathrm{e}(1)$ & $3(3,29)$ \\
\hline & T. verrucosum & - & - & - & - & 1 & - & - & $1(1,09)$ \\
\hline & T. tonsurans & - & 1 & 1 & - & 1 & - & - & $3(3,29)$ \\
\hline & T. rubrum & - & 1 & 3 & - & 3 & - & $\mathrm{c}+\mathrm{e}(1)$ & $8(8,79)$ \\
\hline Microsporum & M. langeronii & 1 & - & - & - & 1 & - & - & $2(2,19)$ \\
\hline Epidermophyton & E. floccosum & - & - & - & - & 2 & - & - & $2(2,19)$ \\
\hline \multirow[t]{2}{*}{ Candida } & $\begin{array}{c}\text { C. albicans :dubliniensis } \\
\text { africana }\end{array}$ & - & 10 & 4 & - & 1 & - & $b+c(3)$ & $18(19,78)$ \\
\hline & C. $s p$. & - & 8 & 2 & - & - & - & - & $10(10,98)$ \\
\hline Trichosporon & T. $s p$. & $* 1$ & 1 & 3 & - & - & - & - & $5(5,49)$ \\
\hline Malassezia & M. furfur & - & - & - & - & - & 26 & - & $26(28,57)$ \\
\hline Fusarium & F. $s p$. & - & - & - & - & - & - & $d+b(1)$ & $1(1,09)$ \\
\hline Neoscytalidium & N. dimidiatum & - & 1 & - & - & - & - & - & $1(1,09)$ \\
\hline \multicolumn{2}{|r|}{ Total } & 2 & 24 & 21 & - & 9 & 26 & 6 & $88(96,70)$ \\
\hline \multicolumn{10}{|c|}{ Co-Infections } \\
\hline \multicolumn{2}{|c|}{ T. mentagrophytes + C. $s p$. } & - & - & 1 & - & - & - & - & $1(1,09)$ \\
\hline \multicolumn{2}{|c|}{ C. albicans + T. rubum } & - & - & - & - & - & - & $\mathrm{c}+\mathrm{e}(1)$ & $1(1,09)$ \\
\hline \multicolumn{2}{|c|}{ C. albicans + A. sp. } & - & 1 & - & - & - & - & - & $1(1,09)$ \\
\hline \multicolumn{2}{|r|}{ Total } & - & 1 & 1 & - & - & - & 1 & $3(3,27)$ \\
\hline
\end{tabular}

ITO: Intertrigo; K: Keratoderma; EP: Epidermophytosis, TV: Tinea versicolor.

* It was a case of white piedra

Table 4: Distribution of species found in the single infections and co-infections depending on the indications in Togo.

\section{Discussion}

Our study was carried out over a period of 1 year in Senegal (February 2019 to February 2020) and 6 months in Togo (October 2019 to March 2020), revealing overall prevalence rates of superficial fungal diseases of $48.67 \%$ and $67.41 \%$ respectively. The prevalence rate found in Togo is very high compared to the figure in Senegal despite the study period half shorter than the one in Senegal. This could be explained, partly, by the quasi-absence of a mycology laboratory in Togo, whereas mycoses are the main reason for consulting a dermatology clinic, according to a study by Kombate K, et al. [9]; and also, partly, by the socio-economic circumstances which makes access to care difficult [10]. Our prevalence, however, is above the $34.2 \%$ reported by Diongue $\mathrm{K}$, et al. [6] between 2011 and 2015 and those reported in other studies: $42.7 \%$ (EMR + CULT +) by Silva-Rocha et al. in northeast Brazil, $36.3 \%$ by Faure-cognet 0 , et al. [12], in the Grenoble region and 32\% by Zahra VL, et al. in Malta [11-13].
Our high prevalence rates reported could be explained by the climatic conditions that prevailed in both countries during the study period. Indeed, hot weather conditions prevailed almost all along our study period in both countries, making it easier for fungi to proliferate. Moreover, a study conducted in Mali (located in the same region as Togo and Senegal) by Coulibaly et al. reported a prevalence of $52.9 \%$, which is in line with our results.

Distribution by gender showed that superficial fungal infections are more frequent among women than men, with prevalences of $61.39 \%$ in Senegal and $58.76 \%$ in Togo. This same trend was reported by Silva-Rocha (64.6\%) in northeast Brazil [11]. This predominance of women is maintained in positive hair and nail samples in Senegal and in positive nail and skin samples in Togo. On the contrary, men were more represented in positive skin samples in Senegal and in positive hair samples in Togo. These observations could be explained by several reasons, namely, attendance of hair 


\section{Open Access Journal of Mycology \& Mycological Sciences}

salons, performance of household chores, and the wearing of tight clothing by either men or women, from one category of samples to another.

A high prevalence of superficial mycoses is observed in Senegal among young patients (under 10 years of age) and in Togo among patients between 31 and 40 years of age. This is probably due in part to their representation in our sample (in Senegal children less than 10 years of age were the most numerous and in Togo patients between 31 and 40 years of age were the most numerous). The other probable reason is that in Senegal we found more ringworm of the scalp (common in infants) and in Togo more Pityriasis versicolor (common in young adults) $[14,15]$. This could also explain the fact that the majority of our positive cases were found in scalp specimens (36.71\%) in Senegal and in skin samples $(68.04 \%)$ in Togo.

Almost the same species of dermatophytes are to be found in both countries except for Trichophyton violaceum found only in Senegal and Trichophyton tonsurans found only in Togo. Since the two countries are located in the same region, it seems logical to find the same species in both countries except for a few. On the contrary, Trichophyton soudanense $(30.37 \%)$ is the predominant dermatophyte in Senegal while in Togo Trichophyton rubrum and Trichophyton mentagrophytes are the predominant species (8.79\% each). We have no previous data on the epidemiology of superficial mycoses in Togo as this is the first study, however in Senegal, previous studies have revealed a predominance of Trichophyton soudanense [4,6]. Trichophyton rubrum and Trichophyton mentagrophytes, following each other in first place, have been reported in other studies [10,12,16-18]. The three predominant dermatophytes found in our study are consistent with the observations made by Binazzi M, et al. [19].

Candida albicans was predominant with a prevalence of $26.96 \%$ in Senegal, which confirms the results reported by Diongue $K$, et al. in Senegal [6], who attributed this observation to the wide distribution of this yeast and its commensal character. In Togo, Malassezia furfur was the most often isolated yeast with a prevalence of $28.57 \%$. This observation is logical considering that tinea versicolor is one of the most common pigmentation disorders in the world, frequently observed in tropical regions with a prevalence of up to $40 \%$ [20].On the other hand, in Senegal it accounted for $1.02 \%$ of superficial mycoses. Indeed, the case of Senegal is quite particular because the majority of our cases of pityriasis versicolor received at the laboratory, claimed to have already made treatments without prior mycological diagnosis, a treatment which in the end did not work. This demonstrates a trend among clinicians to directly prescribe treatments in cases that were not diagnosed as mycological and therefore the patient only arrived at the laboratory when the perceived treatment did not work. We therefore believe that the prevalence rate we found may not reflect the reality of this pathology in the Senegalese population. We also found in our study a case of Trichosporon in the scalp (in Togo). This was a case of white piedra rarely reported in West Africa although most cases occur in tropical regions [14].

The genus Fusarium, which dominates moulds in Senegal, is found in the same proportions as other moulds in Togo. Usually found in soil, they are increasingly involved in human pathology $[21,22]$. They determine, as superficial pathologies, onychomycosis, keratitis and more rarely intertrigos, which are increasingly described in the literature $[6,21,23]$.

\section{Conclusion}

Superficial mycoses remain a real public health issue in Togo and Senegal. The same species are present in both countries with the exception of Trichophyton tonsurans, Trichophyton violaceum and Aspergillus candidus. Dermatophytes are dominated by Trichophyton soudanense in Senegal and Trichophyton rubrum/mentagrophytes in Togo. Yeasts and moulds are dominated by Candida albicans and the genus Fusarium in both countries. Children less than 10 years of age are the most affected in Senegal while adults between 31 and 40 years of age are the most affected in Togo. This study has allowed us to screen the species responsible for superficial mycosis in both countries, which could improve their management.

\section{References}

1. Simonnet C, Berger F, Gantier JC (2011) Epidemiology of superficial fungal diseases in French Guiana: a threeyear retrospective analysis. Med Mycol 49(6): 608-611.

2. Rodrigues ML, Nosanchuk JD (2020) Fungal diseases as neglected pathogens: A wake-up call to public health officials. Plos Negl Trop Dis 14(2): e0007964.

3. Havlickova B, Czaika VA, Friedrich M (2008) Epidemiological trends in skin mycoses worldwide. Mycoses 51(S4): 2-15.

4. Ameen M (2010) Epidemiology of superficial fungal infections. Clin Dermatol 28(2): 197-201.

5. Zhan P, Liu W (2017) The Changing Face of Dermatophytic Infections Worldwide. Mycopathologia 182(1-2): 77-86.

6. Diongue K, Diallo MA, Ndiaye M, Badiane AS, Seck MC, et al. (2016) Causative agents of superficial mycoses isolated in Dakar, Senegal: Retrospective study from 


\section{Open Access Journal of Mycology \& Mycological Sciences}

2011 to 2015. J Mycol Med 26(4): 368-376.

7. Chabasse D, Bouchara JP, De Gentile L, Brun S, Cimon B, et al. (2004) Les dermatophytes. Cahier de formation Bioforma, pp: 159.

8. Bouchara JP, Pihet M, de Gentile L, Cimon B, Chabasse D (2010) Les levures et levuroses. Bioforma Paris, pp: 201.

9. Kombaté K, Saka B, Mouhari-Toure A, Barruet RK, Gnassingbé W, et al. (2014) Skin pathology in the elderly in dermatology in Lome, Togo: study of 325 cases. Pan Afr Med J 18: 151.

10. Zida A, Barro-Traoré $F$, Dera $M$, Bazié $Z$, Niamba $P$, et al. (2015) Epidemiological and etiological aspects of superficial fungal infections among prison inmates in Ouagadougou, Burkina Faso. J Mycol Med 25(2): e73-e79.

11. Silva-Rocha WP, de Azevedo MF, Chaves GM (2017) Epidemiology and fungal species distribution of superficial mycoses in Northeast Brazil. J Mycol Med 27(1): 57-64.

12. Faure-Cognet O, Fricker-Hidalgo H, Pelloux H, Leccia MT (2016) Superficial Fungal Infections in a French Teaching Hospital in Grenoble Area: Retrospective Study on 5470 Samples from 2001 to 2011. Mycopathologia 181(1-2): 59-66.

13. Zahra VL, Gatt P, Boffa MJ, Borg E, Mifsud E, et al. (2003) Characteristics of superficial mycoses in Malta. Int J Dermatol 42(4): 265-271.

14. Bonifaz A, Gómez-Daza F, Paredes V, Ponce RM (2010) Tinea versicolor, tinea nigra, white piedra, and black piedra. Clin Dermatol 28(2): 140-145.
15. Gupta AK, Batra R, Bluhm R, Faergemann J (2003) Pityriasis versicolor. Dermatol Clin 21(3): 413-429.

16. Adefemi SA, Abayomi MA, Abu JM (2010) Superficial fungal infections seen at a tertiary health centre: clinical and mycological studies. West Afr J Med 29(4): 267-270.

17. Di Chiacchio N, Madeira CL, Humaire CR, Silva CS, Fernandes LHG, et al. (2014) Superficial mycoses at the Hospital do Servidor Público Municipal de São Paulo between 2005 and 2011. An Bras Dermatol 89(1): 67-71.

18. Coloe S, Baird R (2010) Dermatophyte infections in Melbourne: Trends from 1961/64 to 2008/09. Australas J Dermatol 51(4): 258-262.

19. Binazzi M, Papini M, Simonetti S (1983) Skin mycoses--geographic distribution and present-day pathomorphosis. Int J Dermatol 22(2): 92-97.

20. Schwartz RA (2004) Superficial fungal infections. Lancet 364(9440): 1173-1182.

21. Bissan AT, Iken M, Doumbia M, Ou-Khedda N, ElAlaoui M, et al. (2017) Fusarioses to Fusarium solani in an immunocompetent and immunocompromised diagnosed in military hospital of Rabat. J Mycol Med 27(3): 382-386.

22. Néji S, Trabelsi H, Cheikhrouhou F, Sellami H, Guidara R, et al. (2013) Fusariosis diagnosed in the laboratory of an UH in Tunisia: Epidemiological, clinical and mycological study. J Mycol Med 23(2): 130-135.

23. Anane S, Chtourou O, Chedi A, Kaouech E, Belhadj S, et al. (2009) Tinea pedis due to Fusarium solani. J Mycol Med 19 (3): 200-202.

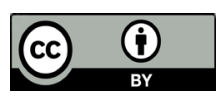

